\title{
On wind-driven electrojets at magnetic cusps in the nightside ionosphere of Mars
}

\author{
M. O. Fillingim ${ }^{1}$, R. J. Lillis ${ }^{1}$, S. L. England ${ }^{1}$, L. M. Peticolas ${ }^{1}$, D. A. Brain ${ }^{1}$, \\ J. S. Halekas ${ }^{1}$, C. Paty ${ }^{2}$, D. Lummerzheim ${ }^{3}$, and S. W. Bougher ${ }^{4}$ \\ ${ }^{1}$ Space Sciences Laboratory, Unverisity of California, Berkeley, CA 94720, USA \\ ${ }^{2}$ School of Earth and Atmospheric Sciences, Georgia Institute of Technology, Atlanta, GA 30332, USA \\ ${ }^{3}$ Geophysical Institute, University of Alaska, Fairbanks, AK 99775, USA \\ ${ }^{4}$ Department of Atmospheric, Oceanic and Space Sciences, University of Michigan, Ann Arbor, MI 48109, USA
}

(Received February 4, 2011; Revised April 15, 2011; Accepted April 19, 2011; Online published March 8, 2012)

\begin{abstract}
Mars has a complex magnetic topology where crustal magnetic fields can interact with the solar wind magnetic field to form magnetic cusps. On the nightside, solar wind electron precipitation can produce enhanced ionization at cusps while closed field regions adjacent to cusps can be devoid of significant ionization. Using an electron transport model, we calculate the spatial structure of the nightside ionosphere of Mars using Mars Global Surveyor electron measurements as input. We find that localized regions of enhanced ionospheric density can occur at magnetic cusps adjacent to low density regions. Under this configuration, thermospheric winds can drive ionospheric electrojets. Collisional ions move in the direction of the neutral winds while magnetized electrons move perpendicular to the wind direction. This difference in motion drives currents and can lead to charge accumulation at the edges of regions of enhanced ionization. Polarization fields drive secondary currents which can reinforce the primary currents leading to electrojet formation. We estimate the magnitude of these electrojets and show that their magnetic perturbations can be detectable from both orbiting spacecraft and the surface. The magnitude of the electrojets can vary on diurnal and annual time scales as the strength and direction of the winds vary. These electrojets may lead to localized Joule heating, and closure of these currents may require field-aligned currents which may play a role in high altitude acceleration processes.
\end{abstract}

Key words: Mars, ionosphere, currents, electrojets.

\section{Introduction}

Mars lacks a globally coherent magnetic field. However, observations have shown that Mars possesses intense and localized crustal magnetic fields (Acuña et al., 1998, 2001). The interaction of the crustal fields with the interplanetary magnetic field (IMF) leads to a complex magnetic topology (Mitchell et al., 2001; Brain et al., 2007). In regions where the crustal fields are nearly radial, they have a tendency to connect to the IMF, forming magnetic cusps. Such cusps provide a conduit for solar wind electrons to interact with the nightside atmosphere. Where this occurs, solar wind electrons should ionize the atmospheric constituents creating a nightside ionosphere. Where crustal fields are horizontal, solar wind electrons do not have access to the atmosphere, and one would expect an absence of ionization, i.e., plasma voids.

Such a complex magnetic topology will create a highly structured nightside ionosphere, particularly in the southern hemisphere where the strongest crustal fields are found. (Electron impact ionization can also be responsible for the formation of a more uniform nighttime ionosphere in the relatively un-magnetized northern hemisphere where solar

Copyright (C) The Society of Geomagnetism and Earth, Planetary and Space Sciences (SGEPSS); The Seismological Society of Japan; The Volcanological Society of Japan; The Geodetic Society of Japan; The Japanese Society for Planetary Sciences; TERRAPUB.

doi:10.5047/eps.2011.04.010 wind electrons have access to the atmosphere along draped solar wind magnetic field lines.) Solar wind electrons have direct access to the atmosphere in the vicinity of magnetic cusps. Electrons are denied access in the vicinity of voids adjacent to cusps. Since the global distribution of cusps is non-uniform (Krymskii et al., 2002) the distribution of night side ionization is likewise expected to be nonuniform, i.e., "patchy."

Recent observations and theoretical work suggest that the nightside ionosphere of Mars is in fact patchy with strong gradients in the ionospheric density. Observations from both Mars Global Surveyor (MGS) (Brain et al., 2006; Halekas et al., 2008) and Mars Express (Lundin et al., 2006a, b) have shown downward-traveling accelerated electrons often collocated with radial magnetic fields at cusps. The accelerated electron spectra have relatively narrow peaks in the electron flux with peak energies from about $100 \mathrm{eV}$ up to a few $\mathrm{keV}$ which are absent in typical photoelectron and solar wind spectra. These spectra are similar in shape to accelerated electron spectra observed in Earth's auroral zone. Enhanced ionization and ionospheric density is expected where these accelerated electrons impact the upper atmosphere.

Data from the Mars Advanced Radar for Subsurface and Ionosphere Sounding (MARSIS) onboard Mars Express have also indicated the presence of structure in the nighttime ionosphere. Enhancements in the maximum electron 
density have been observed on both the dayside (Gurnett et al., 2005, 2008; Duru et al., 2006) and nightside (Kirchner et al., 2006, 2007). The short time duration and the shape of the radar echoes reflecting off of the high density structures indicate that the regions of enhanced density typically have scale sizes of a few tens of $\mathrm{km}$ (Duru et al., 2006). In addition, MARSIS measurements have shown localized increases of the ionospheric density and total electron content (TEC) on the nightside that are generally correlated with vertical magnetic fields in cusp regions (Safaeinili et al., 2007; Mouginot et al., 2008; Němec et al., 2010).

Additionally, Bertaux et al. (2005) reported the first observations of auroral emission at Mars from Mars Express. These and subsequent observations (Leblanc et al., 2008) showed that auroral emission is localized and occurs in the vicinity of magnetic cusps. Leblanc et al. (2008) showed simultaneous observations of auroral-type emissions, precipitating energetic electrons, and increases in the ionospheric TEC. These observations suggest that these phenomena are related leading to a consistent picture with the precipitating electrons causing the enhanced ionization and auroral emission.

Recently, Fillingim et al. (2010) modeled the nightside ionospheric structure using electron observations from MGS as input to an electron transport model. Consistent with observations, they found regions of enhanced ionospheric density at cusps associated with accelerated downward traveling electrons adjacent to regions nearly devoid of ionizaiton (analagous to plasma voids). The modeled horizontal density gradient exceeded $600 \mathrm{~cm}^{-3} \mathrm{~km}^{-1}$.

Fillingim et al. (2010), using their prediced ionospheric structure, also estimated the direction and magnitude of horizontal ionospheric currents driven by neutral thermospheric winds. This estimate relies on the fact that in a particular altitude region of the ionosphere, approximately 110 to $160 \mathrm{~km}$, ions collide with neutrals more often than they gyrate about magnetic field lines (i.e., the ion-neutral collision frequency is much greater than the ion gyrofrequency: $v_{i n} \gg \Omega_{i}$ ) while electrons, with their much higher gyrofrequency, gyrate more frequently than they collide with neutrals $\left(\Omega_{e} \gg v_{e n}\right)$. When an external force is applied to the plasma, the collisional ions move in the direction of the force. The magnetized electrons, on the other hand, drift in a direction perpendicular to both the applied force and the ambient magnetic field. This difference in ion and electon motion leads to currents. The current density, $j$, is calculated by

$$
\mathbf{j}=n q\left(\mathbf{v}_{i}-\mathbf{v}_{e}\right)
$$

where $n$ is the electron and ion number density (assuming charge quasi-neutrality), $q$ is the magnitude of the electronic charge, and $\mathbf{v}_{i}$ and $\mathbf{v}_{e}$ are the ion and electron velocities, respectively. The region where currents can be generated in the ionosphere is refered to as the dynamo region.

Fillingim et al. (2010) solved the equilibrium ion and electron momentum equations in a stationary frame to determine the ion and electron velocities under different driving conditions. Their calculations indicated that, for a neutral wind uniform in both latitude and altitude, the strongest currents were found at magnetic cusps where the iono- spheric density was largest. Similary, the weakest currents were found in regions of low ionospheric density, in plasma voids. This is due in large part to the fact that the current density is directly proportional to the ionospheric density given an approximately constant difference between the ion and electron velocities, as indicated in the above equation. (The difference between the ion and electron velocities is also affected by the magnetic field strength through the gyrofrequency, but their calculations showed that changes in the magnetic field strength had a much smaller effect on the current density than changes in the ionospheric density.)

An important point not considered by Fillingim et al. (2010) is that horizontal gradients in the ionospheric current density should lead to secondary effects. In the terrestrial auroral zone, such secondary effects produce the auroral electrojets (Boström, 1964; Carlson and Egeland, 1995). Auroral precipitation creates a region of enhanced ionization and enhanced conductivity immersed in a background ionosphere of lower conductivity. An applied force, an externally imposed electric field in the terrestrial case, drives ionospheric currents. Currents are stronger where the electron density, hence ionospheric conductivity, is higher. However, in steady state, horizontal currents must be continuous across the boundary between low and high conductivity regions. Neglecting the effect of parallel currents, a transient accumulation of excess charge occurs at the boundary leading to the development of secondary polarization electric fields within the region of high conductivity which then drive secondary currents. Parallel to the polarization electric field, secondary Pedersen currents flow in the direction opposite to the primary (Hall) currents canceling the excess primary current across the boundary. This polarization field also drives secondary Hall currents which act to enhance the primary (Pedersen) currents, creating the auroral electrojets. (In actuality, parallel currents can act to reduce the magnitude of the resulting electrojet.)

Our goal here is to investigate the possibility of wind driven electrojets in the vicinity of cusps in the nightside ionosphere of Mars and to estimate the magnitude of the possible electrojets.

\section{Calculating Ionospheric Currents}

Before we can estimate the magnitude of wind-driven electrojets, we must first calculate the primary currents as done by Fillingim et al. (2010). A brief summary of the these calculations is given below. A more complete description can be found in Fillingim et al. (2010).

To calculate the horizontal ionospheric currents, we start with observations of precipitating electrons obtained from MGS. The top panel of Fig. 1 shows our input electron spectrogram. These data were observed by MGS on 21 April 2001 and have been reported in more detail by Brain et al. (2006). From 02:10 to 02:30 UT, MGS was at an altitude of about nearly $400 \mathrm{~km}$ at a local time of 02 on the night side. During this interval, the spacecraft moved from about $-10^{\circ}$ to $-70^{\circ}$ latitude along the $210^{\circ}$ east meridian while the spacecraft's solar zenith angle varied between $150^{\circ}$ to $120^{\circ}$. Significant variations in the electron energy flux are seen over this interval; two accelerated electron events are seen-one near 02:24 UT and another at 
(a)

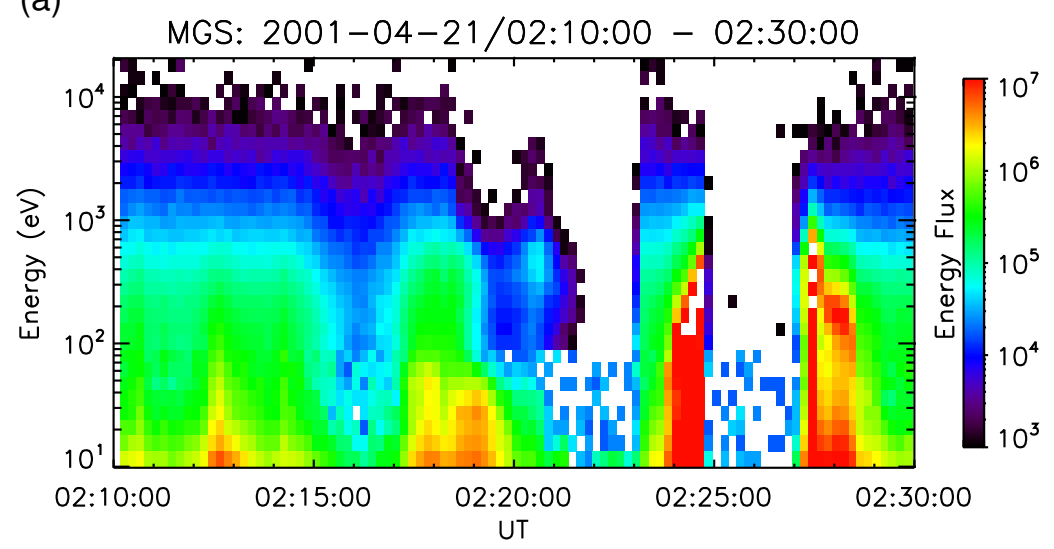

(b)

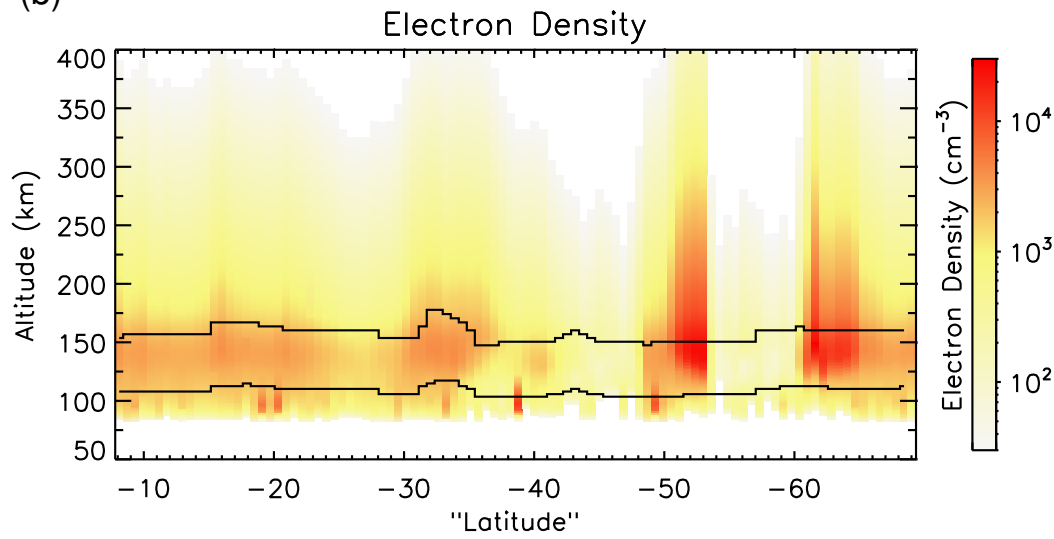

Fig. 1. Input electron energy spectrogram observed by Mars Global Surveyor (top) and the calculated ionospheric electron density due to precipitating electrons (bottom). During the time of observation, MGS was at an altitude of $400 \mathrm{~km}$ and was moving southward from $-10^{\circ}$ to $-70^{\circ}$ latitude along the $210^{\circ}$ East longitude meridian. The spacecraft solar zenith angle varied between $100^{\circ}$ and $120^{\circ}$. The calculated electron density is shown as a function of altitude and "latitude." The altitude where $\Omega / v=1$ for both the ions (lower line) and electrons (upper line) is also shown. The $X$-axis shows the latitude of the spacecraft observation rather than the latitude where the magnetic field intersects the surface. Each column represents the electron density along an assumed straight magnetic field line from an altitude of $400 \mathrm{~km}$ down to $50 \mathrm{~km}$.

02:28 UT_-in cusp regions on either side of a plasma void. A second plasma void is centered at 02:22 UT.

To calculate the ionization rate due to the precipitating electrons, we use a modification of the code of Lummerzheim and Lilensten (1994) developed to model electron transport in the auroral ionosphere of Earth. This code uses the discrete-ordinate method to solve the energy degradation and electron transport problem and uses a multi-stream approach to solve for the electron flux as a function of energy and altitude. The electron transport model requires an atmospheric neutral density profile as input which is given by the Mars Thermospheric Global Circulation Model (MTGCM) (e.g., Bougher et al., 2009). The number densities of $\mathrm{CO}_{2}, \mathrm{CO}, \mathrm{O}_{2}, \mathrm{O}$, and $\mathrm{N}_{2}$ from 100 to $250 \mathrm{~km}$ altitude are supplied by the MTGCM. Below $100 \mathrm{~km}$, the density is extrapolated logarithmically and the temperature is extrapolated linearly. Above $250 \mathrm{~km}$, the density is extrapolated assuming diffusive equilibrium and an isothermal profile ( $T=163 \mathrm{~K}$ in this case). The profile used here is from $2.5^{\circ}$ north latitude at a local time of 3 AM under solar medium conditions at equinox. However, the exact choice of seasonal and geographic parameters is not of critical importance since our goal focuses on differences in the ionospheric density and horizontal currents due to different incident spectra.
Additionally, the appropriate cross sections for electron impact for the relevant neutral species must also be included. Currently, the code incorporates over 100 types of elastic and inelastic collisions including dissociations, excitations, and ionizations using cross sections compiled by Lummerzheim and Lilensten (1994) for $\mathrm{O}, \mathrm{O}_{2}$, and $\mathrm{N}_{2}$, and by J. Fox and K. Sung (personal communication, 2001), Liu and Victor (1994), and Itikawa (2002) for $\mathrm{CO}$ and $\mathrm{CO}_{2}$.

Using this model, a vertical profile of the ion production rate is computed for each input electron spectrum shown in the top panel of Fig. 1 (100 total). The peak total ion production rate varies by five orders of magnitude from $0.003 \mathrm{~cm}^{-3} \mathrm{~s}^{-1}$ in a void to $300 \mathrm{~cm}^{-3} \mathrm{~s}^{-1}$ due to an accelerated spectrum in a cusp. The mean and median peak production rates over the entire interval are 13 and $1.9 \mathrm{~cm}^{-3} \mathrm{~s}^{-1}$, respectively.

The ionospheric electron number density can be computed from the total ion production rate (ignoring dynamics and assuming photochemical equilibrium) from the equation

$$
n_{e}(z)=\left(P(z) / \alpha_{\mathrm{eff}}(z)\right)^{1 / 2}
$$

where $P(z)$ is the ion production rate and $\alpha_{\text {eff }}(z)$ is the effective recombination rate. Due to rapid chemical reactions between $\mathrm{CO}_{2}, \mathrm{O}$, and their ions, $\mathrm{O}_{2}{ }^{+}$is the dominant ion in 
the ionosphere of Mars over the altitudes considered here (Fox et al., 1993, 1996; Haider, 1997). The lifetimes of electron-impact produced $\mathrm{CO}_{2}{ }^{+}$and $\mathrm{O}^{+}$ions at altitudes near the ionospheric peak are less than $10 \mathrm{~s}$ and $0.5 \mathrm{~s}$, respectively, before they react to produce $\mathrm{O}_{2}{ }^{+}$. Therefore, for the following calculations, we make the simplying assumption that all ions quickly become $\mathrm{O}_{2}{ }^{+}$. In this case, $\alpha_{\text {eff }}(z)$ is taken to be the dissociative recombination rate of $\mathrm{O}_{2}{ }^{+}$(e.g., Sheehan and St.-Maurice, 2004):

$$
\alpha_{\mathrm{eff}}(z)=1.95 \times 10^{-7}\left(300 / T_{e}(z)\right)^{0.7} \mathrm{~cm}^{3} \mathrm{~s}^{-1}
$$

where $T_{e}$ is the electron temperature. In the absence of electron temperature observations on the night side, we assume that the electron temperature is equal to the dayside electron temperature derived from the in situ Viking lander observations (Hanson et al., 1977). Specifically, we use the dayside electron temperature of Fox (1993) which fits the data to two exponential functions:

$$
\begin{aligned}
& T_{e}=T_{n}, z<130 \mathrm{~km} \\
& T_{e}=700-536 \exp [(130-z) / 65.4], 130<z<180 \mathrm{~km} \\
& T_{e}=4200-3750 \exp [(190-z) / 89.6], z>180 \mathrm{~km}
\end{aligned}
$$

(This is in contrast to Fillingim et al. (2010) who assumed that the nightside electron temperature was equal to the nightside neutral temperature. As noted by Fillingim et al. (2010), the change in the computed peak ionospheric densities using these two electron temperature profiles is about $15 \%$.)

The computed electron density as a function of altitude and "latitude" is shown in the bottom panel of Fig. 1. The latitude shown on the $X$-axis is the latitude of the spacecraft observation rather than the latitude where the magnetic field lines intersect the surface. Rather than a true twodimensional representation of the ionosphere where there can be significant curvature in the magnetic field, each vertical column in the bottom panel of Fig. 1 represents the electron density along an assumed straight magnetic field line from an altitude of $400 \mathrm{~km}$.

Also shown in bottom panel of Fig. 1 are the altitudes at which the collision frequency, $v$, equals the gyro-frequency, $\Omega$, for the ions (upper line) and for the electrons (lower line). Above these altitudes, magnetic effects dominate the particle dynamics; below these altitudes, collisions dominate the dynamics. This transition occurs at about $160 \mathrm{~km}$ for ions and at about $110 \mathrm{~km}$ for electrons. Between these altitudes is the ionospheric dynamo region.

To determine ionospheric currents, we start with a simplified momentum equation assuming steady state conditions and all forces in equilibrium. Additionally, pressure gradient effects are ignored (these were investigated by Fillingim et al. (2010) and found to be negligible in this context) as are the effects of gravity. In the absense of reliable information regarding ionospheric electric fields, these too are ignored.

In this derivation, we only consider non-resonant collisions between the charged particles (ions and electrons) and atmospheric neutrals. We neglect Coulomb collisions, i.e., ion-ion and ion-electron collisions. Ion-electron collisions couple the electron dynamics to the ion motion and can be important under certain circumstances; however, they do not significantly affect the currents at the altitude of the ionospheric peak (e.g., Heelis et al., 1985). The typical electron-ion collision frequency exceeds the electron neutral collision frequency at an altitude of $\sim 160 \mathrm{~km}$. At this altitude, the electrons will respond more to ion motions than to neutral motions. However, since our calculations are done over a region of Mars containing strong crustal magnetic fields, the electrons are already strongly magnetized at this altitude, so collisional effects will be small. Above weakly magnetized regions, for example in the northern hemisphere of Mars, electron-ion collisions may become essential when describing the electron motion.

With the above assumptions in mind, the simplified ion and electron equations of motion reduce to

$$
\begin{array}{r}
q \mathbf{v}_{i} \times \mathbf{B}-m_{i} v_{i n}\left(\mathbf{v}_{i}-\mathbf{u}\right)=0 \\
-q \mathbf{v}_{e} \times \mathbf{B}-m_{e} v_{e n}\left(\mathbf{v}_{e}-\mathbf{u}\right)=0
\end{array}
$$

Next, we compute the horizontal ionospheric currents due to a uniform meridional neutral wind; $u_{X}$ is set to $100 \mathrm{~m} / \mathrm{s}$ northward at all latitudes and altitudes. This is a gross simplification of the actual neutral wind structure chosen to illustrate the neutral wind effect on the ionospheric currents. However, MTGCM calculations by Bougher et al. $(1999,2000)$ have shown that relatively uniform northward neutral winds with speeds up to a few $100 \mathrm{~m} / \mathrm{s}$ can occur on the nightside in the southern hemisphere particularly near equinox and southern summer solstice.

Additionally, we do not consider the effects of the ions on the neutral wind direction or magnitude. Even at the altitude of the peak ionospheric density, the neutral density is larger than the ionospheric density by a factor of $10^{6}$ (the neutral density is $\sim 10^{10} \mathrm{~cm}^{-3}$ while ion and electron densities are $\sim 10^{4} \mathrm{~cm}^{-3}$ ). For the largest ionospheric densities we compute (a few times $10^{4} \mathrm{~cm}^{-3}$ ), the response time of the neutrals to changes in the ion motion is on the order of the Martian rotation period. Therefore, the impact of the ions on the neutral motion can be safely neglected in these calculations.

We further simplify the problem by assuming that the magnetic field is only in the $Z$-direction, i.e., $\mathbf{B}=B_{z}$. This final simplification is, of course, unphysical since the dip angle varies between $50^{\circ}$ and $90^{\circ}$ over this region according to the model of Cain et al. (2003). We make this simplification so that the particle velocities and currents can be expressed analytically and to enhance the physical understanding of the processes giving rise to the currents. In actuality, non-vertical magnetic fields will lead to vertical transport of the plasma which will modify the altitude and height distribution of the ionized layer. With these assumptions, solving for the ion and electron velocities gives

$$
\begin{aligned}
& v_{i x}=m_{i} v_{i n} u_{x} \frac{v_{i n}}{m_{i}} \frac{1}{\left(\Omega_{i}^{2}+v_{i n}^{2}\right)}=\frac{v_{i n}^{2}}{\left(\Omega_{i}^{2}+v_{i n}^{2}\right)} u_{x} \\
& v_{i y}=-\frac{\Omega_{i}}{v_{i n}} v_{i x} \\
& v_{e x}=m_{e} v_{e n} u_{x} \frac{v_{e n}}{m_{e}} \frac{1}{\left(\Omega_{e}^{2}+v_{e n}^{2}\right)}=\frac{v_{e n}^{2}}{\left(\Omega_{e}^{2}+v_{e n}^{2}\right)} u_{x}
\end{aligned}
$$


(a)

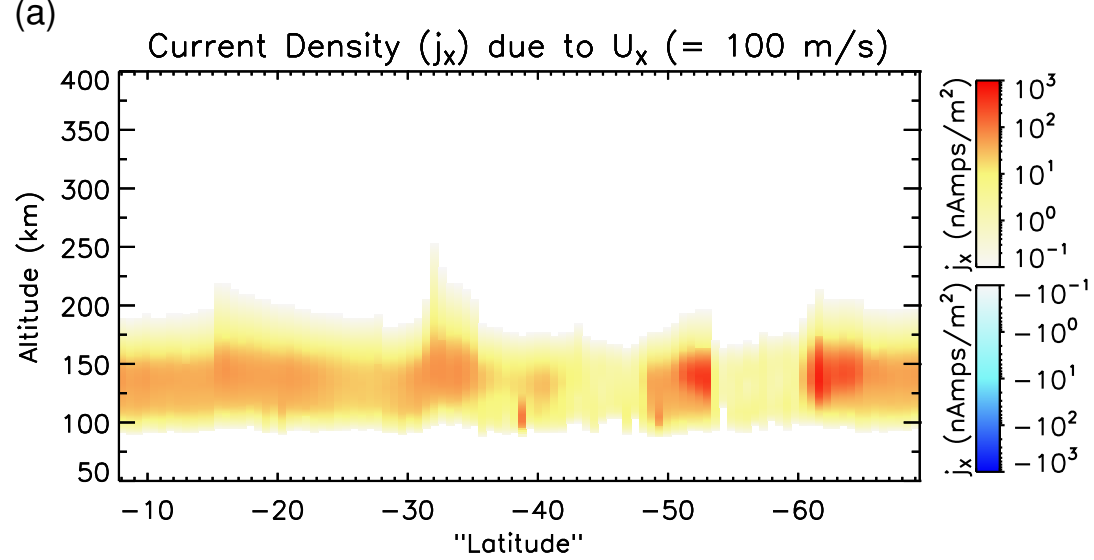

(b)

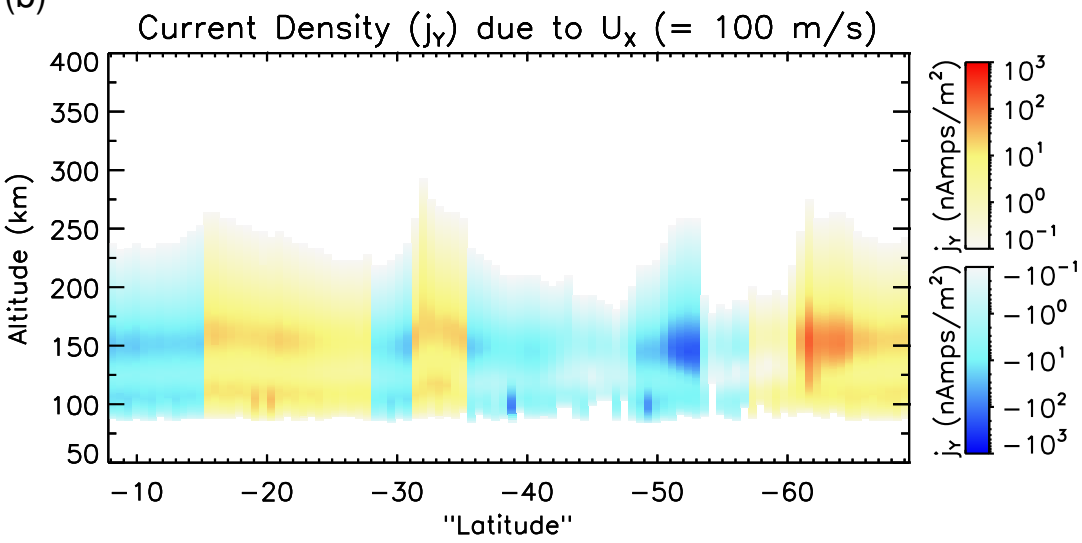

Fig. 2. Calculated $X$ - (top) and $Y$ - (bottom) components of the current density due to a $100 \mathrm{~m} / \mathrm{s}$ neutral wind uniform in latitude and altitude. Pedersen-type currents (parallel to the direction of the applied force) flow in the $X$-direction; Hall-type currents (perpendicular to both the applied force and the magnetic field) flow in the $Y$-direction. In the bottom panel, shades of red indicate westward currents, while shades of blue indicate eastward currents. Abrupt changes in the direction of the $Y$-component of the current are due to changes in the direction of the magnetic field.

$$
v_{e y}=\frac{\Omega_{e}}{v_{e n}} v_{e x}
$$

Once the ion and electron velocities are determined, the latitudinal ( $X$-component) and longitudinal ( $Y$-component) current densities can be computed analytically for all altitudes and latitudes of interest from the current density equation.

Figure 2 shows the $X$ - and $Y$-components of the current density due to a uniform latitudinal neutral wind. The computed currents are highly localized in latitude and altitude. The $X$-component of the current is confined in altitude to the dynamo region (as shown in the bottom panel of Fig. 1). The $X$-component of the current is carried by the collisional ions. In high density regions (in this case, two cusps on either side of a void), ion drag from the northward neutral wind leads to large northward currents. These latitudinal currents are analogous to Pedersen currents in that they flow in the direction of the applied force (the neutral wind) and depend upon the ratio $v /\left(\Omega^{2}+v^{2}\right)$.

The $Y$-component of the current is more extended in altitude, especially at high altitudes. At higher altitudes, $\sim 150 \mathrm{~km}$ at the top of the dynamo region, the $Y$ current is carried by magnetized ions drifting in the $\mathbf{F} \times \mathbf{B}$ direction where $\mathbf{F}=F_{x}=m_{i} v_{i n} u_{x}$. Where $\mathbf{B}$ is positive, i.e., pointing upward from the surface, the $Y$-component of the current is negative, i.e., westward, since the direction of $F_{x} \times B_{z}$ is in the $-Y$ direction. Similarly, where $\mathbf{B}$ is negative (downward), the $Y$-component of the current, $j_{y}$, is positive (eastward). In the bottom panel of Fig. 2, the currents change from positive to negative, eastward to westward, where the sign of $\mathbf{B}$ changes from negative to positive, downward to upward. Again, currents are strongest where ionospheric densities are largest. These currents are Halllike in that they flow perpendicular to the applied force and depend upon the ratio $\Omega /\left(\Omega^{2}+v^{2}\right)$. Since the direction of the magnetic field on each side of the void changes direction, the longitudinal current on each side of the void also changes direction, being directed eastward (negative) near $-52^{\circ}$ latitude and westward (positive) more poleward near $-61^{\circ}$.

At the bottom of the dynamo region, at altitudes $\sim 100 \mathrm{~km}$, the $Y$ current is carried by drifting electrons. The direction of the current is the same as that carried by the ions since electrons drifting in the $-\mathbf{F} \times \mathbf{B}$ direction carry a current in the $\mathbf{F} \times \mathbf{B}$ direction. At higher altitudes, the electron current decreases rapidly since the force acting on the electrons, which is proportional to the electron-neutral collision frequency, $v_{e n}$, decreases. There is a relative minimum in the $Y$ current near $125 \mathrm{~km}$ altitude (where the $X$ currents are strongest) between the higher altitude ion current and the lower altitude electron current. 
(a)

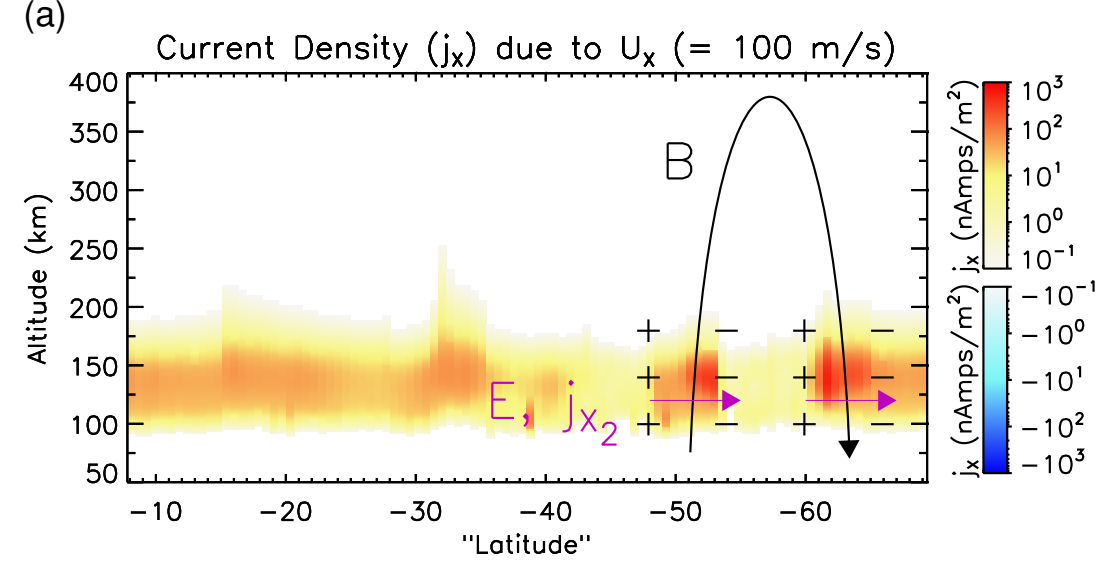

(b)

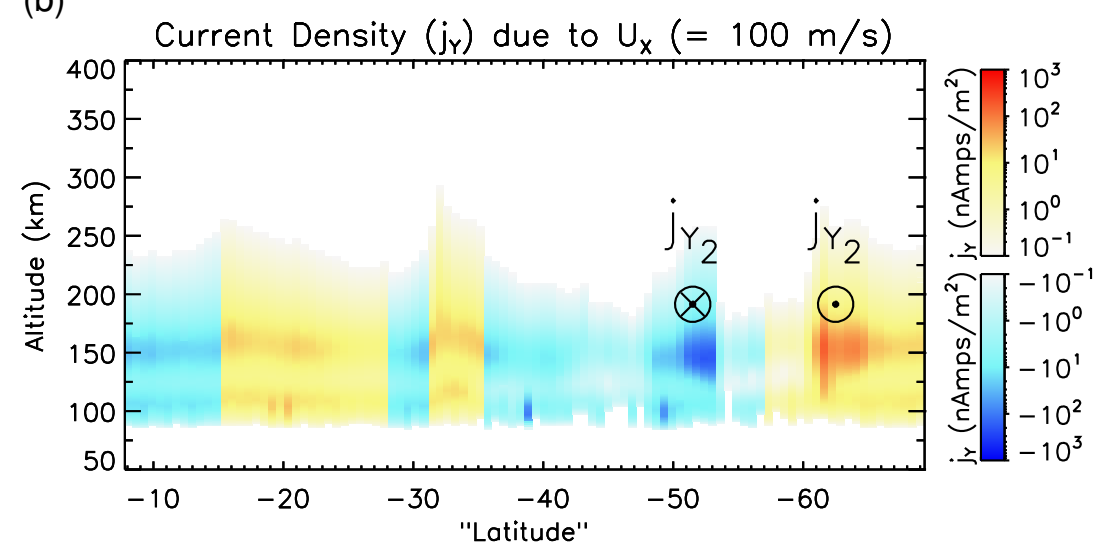

Fig. 3. Same as Fig. 2 overlaid by diagrams of the directions of the secondary polarization electric fields and secondary currents.

\section{Electrojet Estimations}

The above calculations show clear horizontal gradients in the ionospheric current density. These gradients are caused by variations in the spectra of precipitating electrons which leads to variations in the ionospheric density produced by electron impact ionization. As mentioned in Introduction, horizontal gradients in the ionospheric current density will lead to secondary electric field and secondary currents.

Again, in steady state and in the absence of parallel currents, horizontal currents must be continuous across boundaries between regions of low and high density and conductivity. In our case, if we assume uniformity in the longitudinal $(Y)$ direction (the longitudinal scale size of the magnetic cusps in the southern hemisphere is much larger than their latitudinal scale size), the latitudinal component of the current density, $j_{x}$, must be continuous. Similar to the terrestrial auroral case, charge tends to accumulate at the edges of the high conductivity regions (i.e., the high density cusps in the case of Mars). This creates a secondary polarization electric field. In the case considered here, the secondary polarization electric field points southward in the $-X$ direction. This electric field drives southward Pedersen currents, $j_{x_{2}}$, which partially cancel the strong northward currents in the vicinity of the cusps leading to current continuity in the $X$ direction across the cusp-void boundaries. The secondary polarization electric field also drives secondary Hall currents in the $Y$ direction, $j_{y_{2}}$. The secondary electric field-driven Hall currents act to enhance the primary wind-driven currents in the $Y$ direction. Figure 3 shows a schematic of this configuration superposed on the primary currents calculated above.

In order to estimate the magnitude of the secondary currents, we assume that at each altitude $j_{x}$ is continuous in latitude and is equal to its minimum value at that altitude, $j_{x}^{\min }$.

Therefore, at each altitude, can we compute the secondary polarization electric field, $E_{x}$, at each latitude point:

$$
\begin{aligned}
& j_{x_{2}}=j_{x}^{\min }-j_{x}=\sigma_{\mathrm{P}} E_{x} \\
& E_{x}=\left(j_{x}^{\min }-j_{x}\right) / \sigma_{\mathrm{P}}
\end{aligned}
$$

where $\sigma_{\mathrm{P}}$ is the Pedersen conductivity and is given by

$$
\sigma_{\mathrm{P}}=n_{e} q^{2}\left[\frac{1}{m_{e}} \frac{v_{e n}}{\Omega_{e}^{2}+v_{e n}^{2}}+\frac{1}{m_{i}} \frac{v_{i n}}{\Omega_{i}^{2}+v_{i n}^{2}}\right]
$$

Since we assume all ions are $\mathrm{O}_{2}{ }^{+}, m_{i}$ is the $\mathrm{O}_{2}{ }^{+}$ion mass.

The secondary current in the $Y$ direction, $j_{y_{2}}$, is then

$$
j_{y_{2}}=\sigma_{\mathrm{H}} E_{x}=\sigma_{\mathrm{H}}\left(j_{x}^{\min }-j_{x}\right) / \sigma_{\mathrm{P}} \approx-\sigma_{\mathrm{H}} / \sigma_{\mathrm{P}} j_{x}
$$

The final term on the right is a reasonable approximation since the minimum $X$-directed current density at each altitude, $j_{x}^{\min }$, is typically located in the low density plasma void and hence quite small. (The negative sign is simply a product of our geometry: for a positively directed $j_{x}, j_{y_{2}}$ is 
(a)

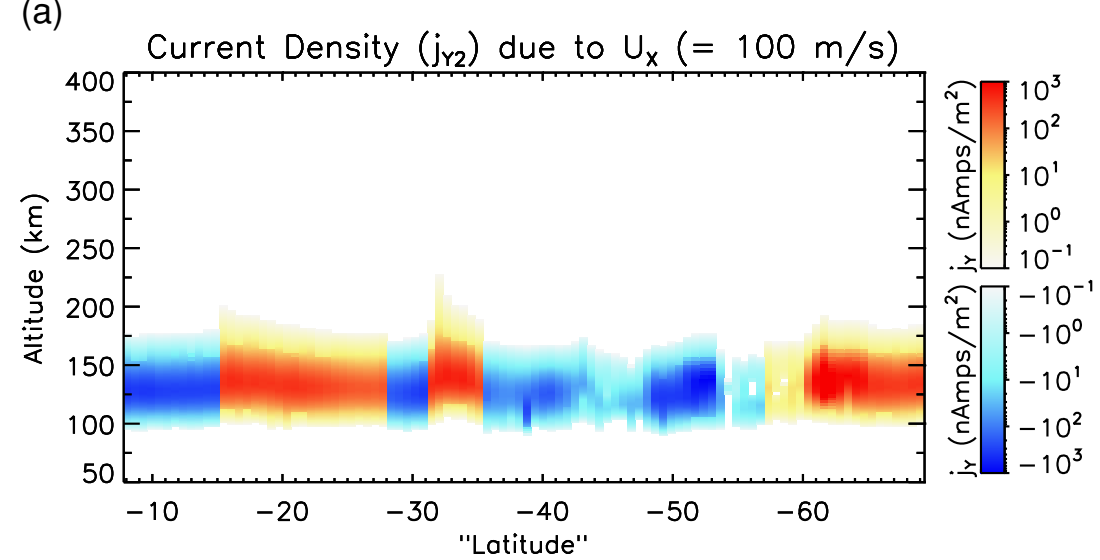

(b)

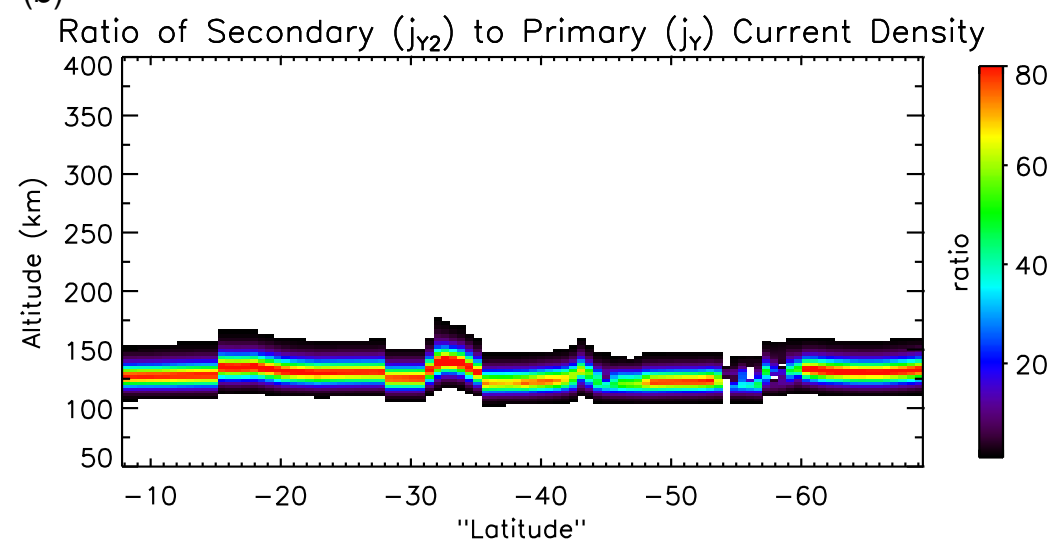

Fig. 4. The secondary current density in the $Y$ direction, $j_{y_{2}}$ (top), and the ratio of the secondary to primary current densities in the $Y$ direction, $j_{y_{2}} / j y$ (bottom). The direction of the secondary $Y$ currents is the same as the primary $Y$ currents leading to an enhancement in the current density.

in the negative $Y$ direction for a magnetic field in the positive $Z$ direction.) $\sigma_{\mathrm{H}}$ is the Hall conductivity given by

$$
\sigma_{\mathrm{H}}=n_{e} q^{2}\left[\frac{1}{m_{e}} \frac{\Omega_{e}}{\Omega_{e}^{2}+v_{e n}^{2}}-\frac{1}{m_{i}} \frac{\Omega_{i}}{\Omega_{i}^{2}+v_{i n}^{2}}\right]
$$

The top panel of Fig. 4 shows the calculated secondary current, $j_{y_{2}}$, computed with a non-zero $j_{x}^{\mathrm{min}}$. It is clear from comparisons with Fig. 3 that the direction of the secondary $Y$ current is the same as that of the primary $Y$ current; however, the magnitude of the secondary current is much larger. Additionally, the secondary current is confined to a narrower altitude range similar to that of the primary $X$ current (top panel of Figs. 2 and 3).

Unlike the primary currents, $j_{x}$ and $j_{y}$, which are carried mainly by ion motion, $j_{y_{2}}$ is carried almost exclusively by electrons. The neutral wind force, $m v u_{x}$, is proportional to the particle mass. The force on and the current carried by the ions is much larger than that for the electrons. However, the magnitude of the electric force, $q E_{X}$, is the same on both ions and electrons. In the dynamo region, the Hall conductivity of the electrons is several hundred times larger than the Hall conductivity of the ions leading to an electron dominated current.

Next we compute the factor by which the $Y$ current has been enhanced. The bottom panel of Fig. 4 shows the ratio of the secondary current to the primary current, $j_{y_{2}} / j_{y}$. The current is greatly enhanced in a relatively narrow altitude region. This enhancement occurs at altitudes where the secondary current, $j_{y_{2}}$, is strongest (cf. the top panel of Fig. 4) and where the primary current, $j_{y}$, has a relative minimum (cf. the bottom panel of Fig. 2). The strongest enhancements occur in regions of high ionospheric density, at the cusps. The current is only weakly enhanced in voids (cf. Fig. 1). This is because the primary $X$ current, $j_{x}$, is weak and approximately equal to the minimum $X$ current, $j_{x}^{\mathrm{min}}$. Therefore, the polarization electric field, $E_{x}$, is quite small in voids. Since $j_{x}$ is large at the cusps, $E_{x}$ is also large in the vicinity of the cusps which leads to large secondary $Y$ currents.

Mathematically, this ratio can be expressed as

$$
\frac{j_{y_{2}}}{j_{y}}=\frac{\left(j_{x}^{\min }-j_{x}\right)}{j_{y}} \frac{\sigma_{\mathrm{H}}}{\sigma_{\mathrm{P}}} \approx \frac{-j_{x}}{j_{y}} \frac{\sigma_{\mathrm{H}}}{\sigma_{\mathrm{P}}}=\left(\frac{\sigma_{\mathrm{H}}}{\sigma_{\mathrm{P}}}\right)^{2}
$$

(Again, for positive $j_{x}, j_{y}$ and $j_{y_{2}}$ are negative, so this ratio is a positive quantity.) This same relation can be derived for the case of Earth's auroral electrojet (e.g., Carlson and Egeland, 1995).

In the dynamo region of the nightside of Mars, we compute the typical maximum of the ratio of the Hall to Pederson condutivites to be about 9 . Therefore, from the above equation, the typical maximum of the ratio of the secondary $Y$ current to the primary $Y$ current is approximately 80 , as shown in the bottom panel of Fig. 4. This maximum is found at cusps and at most other latitudes where signifi- 
(a)

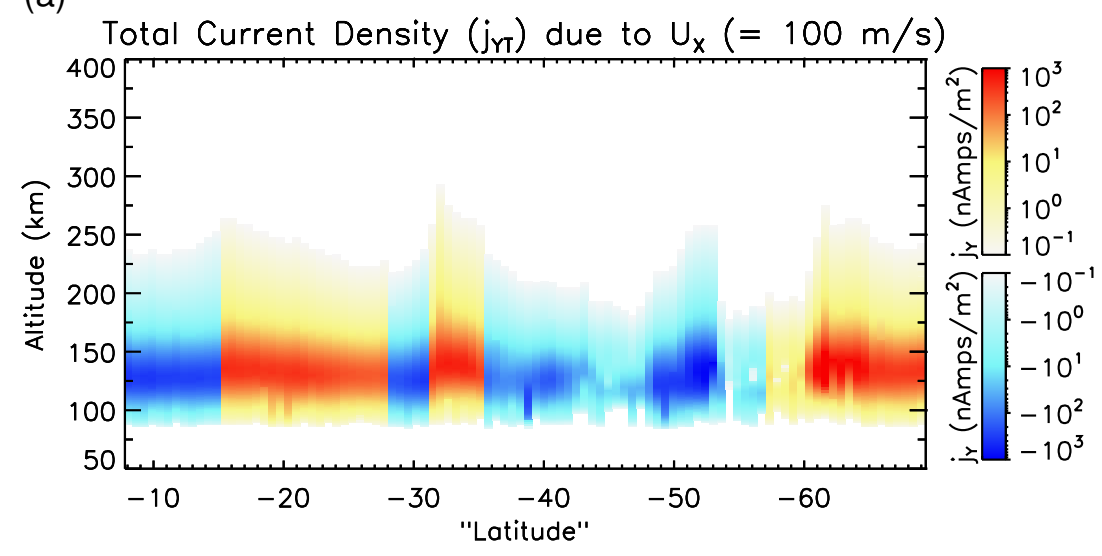

(b)

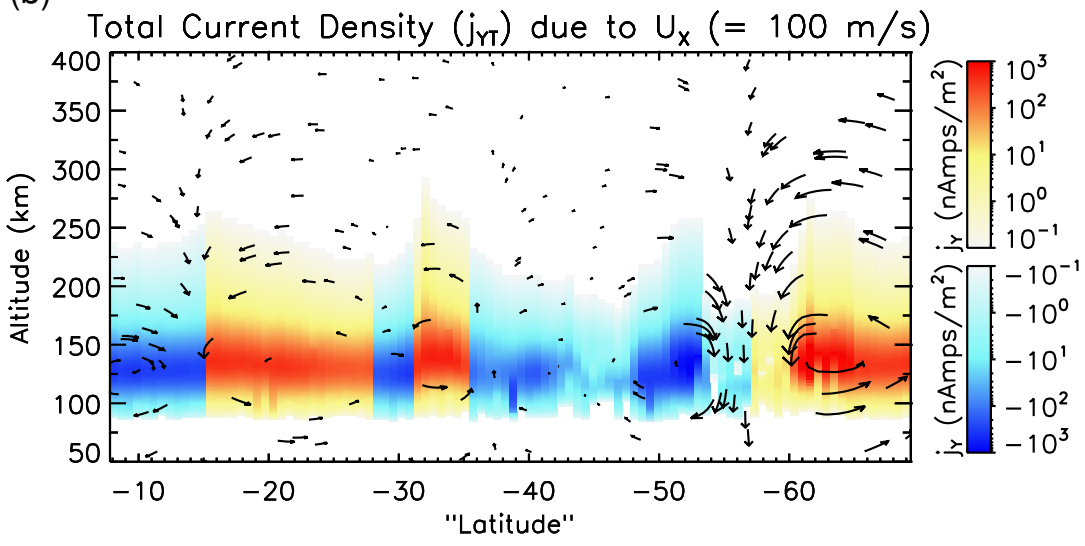

Fig. 5. The total current density in the $Y$ direction, $j_{y_{T}}$ (top), and the total current density overlaid by arrows representing the strength and direction of the magnetic field produced by the current (bottom).

cant secondary currents are calculated while much smaller enhancements are seen in voids.

Since we calculate a large enhancement in the ionospheric currents at magnetic cusps associated with increased conductivity due to precipitating electrons, we term these enhanced currents electrojets in analogy with similar phenomena on Earth.

The top panel of Fig. 5 shows the total current density in the $Y$ direction, $j_{y_{T}}=j_{y}+j_{y_{2}}$. The intense secondary currents dominate the $Y$ current, but the weaker primary $Y$ currents are present over a wider altitude range.

Using the Biot-Savart law, we calculate the magnetic field generated by the total $Y$ current. Example magnetic lines of force are shown in the bottom panel of Fig. 5. The arrows point in the direction of the force and the length of the curve segments indicate the strength of the field. Since the maximum $Y$ current densities occur in the cusps at latitudes near $-52^{\circ}$ and $-62^{\circ}$, the maximum generated magnetic field also occurs in this region between latitudes $-50^{\circ}$ and $-65^{\circ}$. In the vicinity of the cusps, we can then compare the strength of the generated field to that of the ambient field as given by the model of Cain et al. (2003). At $400 \mathrm{~km}$ altitude in this latitude range, the magnitude of the current generated magnetic field is about $10 \mathrm{nT}$. The ambient magnetic field due to crustal sources is on the order of $100 \mathrm{nT}$. The current generated field is about $10 \%$ of the ambient field. At $150 \mathrm{~km}$ altitude, the current generated field is $\sim 50 \mathrm{nT}$ while the ambient field is $\sim 500 \mathrm{nT}$. Again, the current generated field is about $10 \%$ of the ambient field. At the surface, the magnetic field is estimated to be $\sim 1000 \mathrm{nT}$ (using the model of Cain et al. (2003)). The calculated current generated field is $\sim 10 \mathrm{nT}$, about $1 \%$ of the surface field. This percent change is approximately the same as that seen in the terretrial auroral zone: a surface magnetic purturbation of $\sim 500 \mathrm{nT}$ for moderate auroral activity in an ambient field of $\sim 50,000 \mathrm{nT}$. Based on these calculations, the magnetic signatures of cusp electrojets should be observable from low altitude orbiting spacecraft or from a magnetometer on the surface of Mars.

At the edges of the electrojets, there is a strong vertical component to the current generated perturbation magnetic field which can enhance or decrease the ambient crustal field. In this work, with a uniform northward driving neutral wind, it appears that current generated magnetic fields enhance the crustal magnetic field northward of the electrojet and decrease the crustal field southward of the electrojet. This is particularly evident for the two regions of strongest currents near $-51^{\circ}$ and $-63^{\circ}$ latitude. South of $-57^{\circ}$, the crustal fields are generally vertically downward. The polarity switches to generally vertically upward northward of $-57^{\circ}$ (as shown in the top panel of Fig. 3). (As noted earlier, it is the change in polarity of the magnetic field which causes the direction of the $Y$ current to change.) Southward of the electojet centered near $-63^{\circ}$, the crustal field is pre- 
dominantly downward while the current generated field has an upward component decreasing the ambient crustal field. In the void immediately northward of the electrojet, near $-59^{\circ}$, both the ambient crustal field and the current induced field have vertically downward components. (Between the cusps/electrojets, in the void where the crustal magnetic field changes polarity, there is a also significant horizontal component of the field which we ignore in these calculations. This is discussed in more detail in Section 5) Northward of the crustal magnetic polarity reversal at $-57^{\circ}$ and southward of the electrojet centered near $-51^{\circ}$, the crustal field has an upward vertical component while the current generated field is predominantly downward, again decreasing the ambient field. At the northern edge of the electrojet, near $-48^{\circ}$, both the crustal field and the current generated field have vertically upward components. This trend continues for other regions of enhanced current shown in the bottom panel of Fig. 5, particularly near crustal magnetic field polarity reversals where the vertical component of the current generated field is strongest.

A brief gedanken experiment suggests that this trend will reverse if the driving neutral wind direction is reversed; for a uniform southward neutral wind, the current generated magnetic field will decrease the ambient crustal field north of the electrojets and enhance the ambient field south of the electrojets. As discussed in Section 5, these current generated perturbation magnetic fields may change the magnetic topology and thus significantly impact the ionospheric electrodynamics.

\section{Variability of the Electrojet}

Thus far we have estimated the magnitude of wind driven electrojets using a fixed set of parameters that specify a particular ionospheric conductivity profile and neutral wind profile. As the horizontal ionospheric conductivity structure changes or as the neutral wind speed or direction changes, the electrojet intensity would also be expected to change.

There are several periodic variations that could affect the intensity of the electrojets. There is a strong diurnal variation in the ionospheric conductivity. In sunlight, the horizontal ionospheric conductivity gradients are weaker. In this case, one would expect the $X$ component of the current to be more continuous, i.e., $j_{x}^{\min }$ would be larger since ample ionization produced by solar EUV would be present in the closed field regions (voids on the nightside) to carry current. Weaker conductivity gradients would be expected to lead to weaker electrojet intensities. However, as recently pointed out by Opgenoorth et al. (2010), strong ionospheric conductivity gradients can still be present on the dayside between adjacent strongly magnetized and weakly magnetized regions. They suggested that secondary effects (including electrojets and/or field aligned currents) could be electrodynamically important on the dayside due to such conductivity gradients. On the nightside, conductivity gradients are supported by particle precipiation as well as changes in the magnetic field intensity.

Global circulation models have shown that wind patterns change with local time (e.g., Bougher et al., 2000) leading to another source of diurnal variation in the expected electrojet intensity. The results of Bougher et al. (2000) sug- gested that generally northward winds are expected in the magnetized southern hemisphere pre-midnight. This is the configuration we have adopted for the calculations shown here.

However, Bougher et al. (2000) also showed that in the post-midnight sector, the thermospheric neutral winds are generally directed westward in the southern hemisphere. Although we do not show calculations for a westward directed neutral wind, our examination indicates that secondary polarization electric fields and currents can be generated, but that the direction of the secondary currents is opposite to that of the primary currents. A westward, longtitudinal wind will drive westward, longitudinal currents via ion drag. North-south, latitudinal currents will also be created in the $\mathbf{F} \times \mathbf{B}$ direction (see Fillingim et al., 2010). Particle accumulation is still expected at the cusp-void boundaries where the conductivity changes setting up secondary polarization electric fields. Hall currents driven by these electric fields will flow in the $Y$-direction; however, the geometry is such that the Hall currents flow in the direction opposite to the primary wind-driven $Y$ currents. That is, rather than enhancing the primary current, the secondary currents can act to decrease the primary current. In such a case, we would expect weaker currents rather than an enhanced electrojet.

In additional to diunal variations, work by Bougher et al. $(1999,2000)$ suggest that there are also seasonal variations in the neutral wind patterns. During equinox and southern summer solstice, northward thermospheric winds are present in the southern hemisphere on the nightside (at least at some local times). During northern summer solstice, neutral winds are generally eastward in the southern hemisphere on the nightside. Again, we expect the secondary currents due to eastward winds to oppose the primary winddriven currents, leading to weaker currents rather than an electrojet.

Finally, the effects of the solar wind and interplanetary magnetic field (IMF) may lead to non-periodic variations in the electrojet intensity. The solar wind pressure and IMF direction can impact the magnetic topology on the nightside, influencing where solar wind particles have access to the atmosphere (Brain, 2006). Additionally, the solar wind pressure and IMF direction are also related to the presence of accelerated precipitating electrons (Brain et al., 2006). Therefore, as the solar wind and IMF change, the location of conductivity gradients can change as well as the strength of the gradients. Such variations in the location and strength of conductivity gradients will presumably influence the intensity of the secondary currents in a complex and dynamic fashion.

\section{Discussion}

There are several caveats and assumptions to keep in mind when considering these results. The electron transport model used in these calculations does not include gradients in the magnetic field. Magnetic field lines are assumed to be straight with a constant dip angle and magnitude. At Mars, where the scale length of changes in the magnetic field can be relatively small, this is a poor assumption. We try to minimize the impact of this assumption by choosing a dip 
angle of the magnetic field that matches the dip angle given by the crustal magnetic field model of Cain et al. (2003) near $150 \mathrm{~km}$, the altitude of greatest ionization. As recently shown by Lillis et al. (2009), strong magnetic gradients can reflect a significant portion of the downward-travelling electrons and can greatly affect the ionization profile. However, if the down-going spectrum is isotropic, the electron flux remains approximately constant with altitude because loss of electrons by magnetic reflection is balanced by the decrease in area of the flux tube through which the electrons travel; only the area upon which the electrons precipitate will decrease. (However, Lillis et al. (2009) showed that, by including altitude dependent scattering effect of the atmosphere, magnetic gradients can also impact the ionization profile due to isotropic distrubutions changing the peak ionization rate by $\sim 20 \%$ between the zero crustal field case and the strongest crustal field gradient they considered.) Brain et al. (2006) showed that throughout most of the interval shown in the top panel of Fig. 1 electron pitch angles observed by MGS were approximately isotropic, so the effects of neglecting magnetic gradients should be minimal.

As mentioned earlier, for analytical simplicity we assume that the magnetic field is vertical, i.e., $\mathbf{B}=B_{z}$, when calculating the horizontal ionospheric currents and secondary effects. Given the small scale structure of the magnetic field, this is also a poor assumption. This simplification was made in order to focus on horizontal currents and secondary effects in the ionosphere and to try to understand the physical processes which may be generating them. An $X$ - or $Y$ component of the magnetic field will result in a $Z$ - (vertical) component of the current density giving rise to more complicated three-dimensional current systems. In the vicinity of magnetic cusps associated with strong crustal magnetic sources, the magnetic field orientation is dominantly radial. In these local regions, the processes we describe here may occur. However, far from strong, radial crustal fields, our results may not be valid.

In this work, we have neglected the effects of external (e.g., magnetospheric) electric fields. In the terrestrial auroral ionosphere, magnetospheric electric fields are dominant drivers of ionospheric currents. Could this also be the case in the ionosphere of Mars? In the absence of low altitude electric field measurments, we choose to focus on winddriven effects, leaving the possibility of horizontal currents and electrojets driven by external electric fields to be addressed by future observations.

In our calculation of secondary polarization electric fields and currents, we have also ignored field aligned currents. The presence of field aligned currents have been infered from observations of perturbations in the magnetic field near magnetic cusps (Brain et al., 2006; Halekas et al., 2006). The magnitude of these currents are $\sim 0.5$ to $1 \mu \mathrm{A}$ $\mathrm{m}^{-2}$. The theoretical calculations by Dubinin et al. (2008) are in good agreement with these observations. By removing some of the charge accumulating at the interface of the high and low conductivity regions, parallel currents will act to decrease the polarization electric field and the resulting secondary currents. The addition of field aligned currents would result in three dimensional current system in which one could imagine currents flowing along field lines into the ionosphere along the boundary of a cusp, flowing horizontally throught the ionosphere in the cusp itself, and flowing up out of the ionosphere along magnetic field lines at the other side of the cusp that bound a plasma void. This is somewhat analogous to Region 1 and Region 2 currents in the terrestrial auroral zone (Iijima and Potemra, 1976) where currents flow into the ionosphere, across the auroral zone, and then back out of the ionosphere. On Mars, these currents could then re-enter the ionosphere on the other side of the void, flow horizontally through another cusp region before exiting the ionosphere as a field aligned current again. Again, in analogy with Earth's auroral zone, such field aligned currents can decrease, but do not nullify, the magnitude of the electrojets.

Finally, even though we have estimated the night side horizontal ionospheric currents, we have not selfconsistently considered the effects of these currents. The currents themselves will generate magnetic fields (as shown in Fig. 5) and perturb the ambient magnetic field. Here, we estimate that the current generated magnetic fields can be $\sim 10 \%$ of the abmient field. Earlier estimations by Withers et al. (2005) suggested that the magnetic field due to ionospheric currents could be up to $40 \%$ of the ambient magnetic field. Since the presence of ionospheric currents will change the magnetic topology, the effects of the currents must be included self-consistently to accurately describe the ionospheric electrodynamics.

\section{Conclusion}

The complex magnetic topology at Mars allows solar wind and magnetotail electrons to ionize the nightside atmosphere in limited regions, mainly near cusps, forming a patchy nightside ionosphere. Neutral winds can drive ionospheric currents at altitudes where ions are collisionally coupled to the neutral atmosphere while electrons are magnetized. Inhomogeneities in the ionospheric conductivity can lead to polarization electic fields and secondary ionospheric currents which can reinforce primary currents forming electrojets. Given the simplifying assumuptions, these calculations of eletroject magnitudes are undoubtedly an overestimate. However, through analogy with the terrestrial auroral eletrojects, they do provide a rationale for the possible existince of secondary electrodynamic effects associated with inhomogeneities in ionospheric density, magnetic field, and conductivity. The magnetic signatures of the electrojets can be significant $(\sim 10 \%)$ and are predicted to be observable from low altitude orbit $(<400 \mathrm{~km})$ as well as from the surface. Given the known and predicted variabilities in the ionospheric conductivity gradients and neutral winds, the electrojet intensity should likewise be variable in periodic and non-periodic ways.

Acknowledgments. This work was supported in part by NASA grants NNX06AD97G, NXX08AK94G, and NNX09AD43G. SWB was supported by NASA grant NNG04GJ94G.

Guest editor M. Yamauchi thanks L. Andersson and an anonymous reviewer in evaluating this paper.

\section{References}

Acuña, M. H. et al., Magnetic field and plasma observations at Mars: Initial results of the Mars Global Surveyor mission, Science, 279, 1676-1680, 
1998.

Acuña, M. H. et al., Magnetic field of Mars: Summary of results from the aerobraking and mapping orbits, J. Geophys. Res., 106, 23,403-23,417, 2001.

Bertaux, J.-L., F. Leblanc, O. Witasse, E. Quemerais, J. Lilensten, S. A. Stearn, B. Sandel, and O. Koralev, Discovery of an aurora on Mars, Nature, 435, 790-794, 2005.

Boström, R., A model of the auroral electrojets, J. Geophys. Res., 69, 4983-4999, 1964.

Bougher, S. W., S. Engel, R. G. Roble, and B. Foster, Comparative terrestrial planet thermospheres 2 . Solar cycle variation of global structure and winds at equinox, J. Geophys. Res., 104, 16,591-16,611, 1999.

Bougher, S. W., S. Engel, R. G. Roble, and B. Foster, Comparative terrestrial planet thermospheres 3 . Solar cycle variation of global structure and winds at solstices, J. Geophys. Res., 105, 17,669-17,692, 2000.

Bougher, S. W., T. M. McDunn, K. A. Zoldak, and J. M. Forbes, Solar cycle variability of Mars dayside exospheric temperatures: Model evaluation of underlying thermal balances, Geophys. Res. Lett., 36, L05201, 2009.

Brain, D. A., Mars Global Surveyor measurements of the Martian solar wind interaction, Space Sci. Rev., 126, 77-112, 2006.

Brain, D. A., J. S. Halekas, L. M. Peticolas, R. P. Lin, J. G. Luhmann, D. L. Mitchell, G. T. Delory, S. W. Bougher, M. H. Acuña, and H. Rème, On the origin of aurorae on Mars, Geophys. Res. Lett., 33, L01201, 2006.

Brain, D. A., R. J. Lillis, D. L. Mitchell, J. S. Halekas, and R. P. Lin, Electron pitch angle distributions as indicators of magnetic field topology near Mars, J. Geophys. Res., 112, A09201, 2007.

Cain, J. C., B. B. Ferguson, and D. Mozzoni, An n $=90$ internal potential function of the Martian crustal magnetic field, J. Geophys. Res., 108, 5008, 2003.

Carlson, H. C., Jr. and A. Egeland, The aurora and the auroral ionosphere, in Introduction to Space Physics, edited by Kivelson, M. G. and C. T. Russell, 568 pp, Cambridge University Press, New York, 1995.

Dubinin, E., G. Chanteur, M. Fraenz, and J. Woch, Field-aligned currents and parallel electric field potential drops at Mars. Scaling from the Earth's aurora, Planet. Space Sci., 56, 868-872, 2008.

Duru, F., D. A. Gurnett, T. F. Averkamp, D. L. Kirchner, R. L. Huff, A. M. Persoon, J. J. Plaut, and G. Picardi, Magnetically controlled structures in the ionosphere of Mars, J. Geophys. Res., 111, A12204, 2006.

Fillingim, M. O., L. M. Peticolas, R. J. Lillis, D. A. Brain, J. S. Halekas, D. Lummerzheim, and S. W. Bougher, Localized ionization patches in the nighttime ionospheric of Mars and their electrodynamic consequences, Icarus, 206, 112-119, 2010.

Fox, J. L., The production and escape of nitrogen atoms on Mars, J. Geophys. Res., 98, 3297-3310, 1993.

Fox, J. L., J. F. Brannon, and H. S. Porter, Upper limits to the nightside ionosphere of Mars, Geophys. Res. Lett., 20, 1339-1342, 1993.

Fox, J. L., P. Zhou, and S. W. Bougher, The Martian thermosphere/ionosphere at high and low solar activities, Adv. Space Res., 17, 203-218, 1996.

Gurnett, D. A., D. L. Kirchner, R. L. Huff, D. D. Morgan, A. M. Persoon, T. F. Averkamp, F. Duru, E. Nielsen, A. Safaeinili, J. J. Plaut, and G. Picardi, Radar soundings of the ionosphere of Mars, Science, 310, 1929-1933, 2005.

Gurnett, D. A. et al., An overview of radar soundings of the Martian ionosphere from the Mars Express spacecraft, Adv. Space Res., 41, 1335-1346, 2008.

Haider, S. A., Chemistry on the nightside ionosphere of Mars, J. Geophys. Res., 102, 407-416, 1997.

Halekas, J. S., D. A. Brain, R. J. Lillis, M. O. Fillingim, D. L. Mitchell, and R. P. Lin, Current sheets at low altitudes in the Martian magnetotail, Geophys. Res. Lett., 33, L13101, 2006.

Halekas, J. S., D. A. Brain, R. P. Lin, J. G. Luhmann, and D. L. Mitchell, Distribution and variability of accelerated electrons at Mars, Adv. Space Res., 41, 1347-1352, 2008.

Hanson, W. B., S. Sanatani, and D. R. Zuccaro, The Martian ionosphere as observed by the Viking retarding potential analyzers, J. Geophys. Res., 82, 4351-4363, 1977.

Heelis, R. A., J. F. Vickrey, and N. B. Walker, Electrical coupling effects on the temporal evolution of F-layer plasma structure, J. Geophys. Res., 90, 437-445, 1985.

Iijima, T. and T. A. Potemra, The amplitude distribution of field-aligned currents at northern high latitudes observed by Triad, J. Geophys. Res., 81, 2165-2174, 1976.

Itikawa, Y., Cross sections for electron collisions with carbon dioxide, $J$ Phys. Chem. Ref. Data, 31, 749-767, 2002.

Kirchner, D. L., D. A. Gurnett, A. Safaeinili, D. D. Morgan, R. L. Huff, J. J. Plaut, and G. Picardi, Radar sounding observations of the nightside Martian ionosphere, Geophys. Res. Abs., 8, 05224, 2006.

Kirchner, D. L., D. A. Gurnett, J. D. Winningham, A. Safaeinili, J. J. Plaut, and G. Picardi, Auroral ionization patches on the nightside of Mars, Geophys. Res. Abs., 9, 04627, 2007.

Krymskii, A. M., T. K. Breus, N. F. Ness, M. H. Acuña, J. E. P. Connerney, D. H. Crider, D. L. Mitchell, and S. J. Bauer, Structure of the magnetic field fluxes connected with crustal magnetization and topside ionosphere at Mars, J. Geophys. Res., 107, 1245, 2002.

Leblanc, F. et al., Observations of aurorae by SPICAM ultraviolet spectrograph on board Mars Express: Simultaneous ASPERA-3 and MARSIS measurements, J. Geophys. Res., 113, A08311, 2008.

Lillis, R. J., M. O. Fillingim, L. M. Peticolas, D. A. Brain, R. P. Lin, and S. W. Bougher, Nightside ionosphere of Mars: Modeling the effects of crustal magnetic fields and electron pitch angle distributions on electron impact ionization, J. Geophys. Res., 114, E11009, 2009.

Liu, W. and G. A. Victor, Electron energy deposition in carbon monoxide gas, Astrophys. J., 435, 909-919, 1994.

Lummerzheim, D. and J. Lilensten, Electron transport and energy degradation in the ionosphere: Evaluation of the numerical solution, comparison with laboratory experiments and auroral observations, Ann. Geophys., 12, 1039-1051, 1994.

Lundin, R. et al., Plasma acceleration above Martian magnetic anomalies, Science, 311, 980-983, 2006a.

Lundin, R. et al., Auroral plasma acceleration above Martian magnetic anomalies, Space Sci. Rev., 126, 333-354, 2006 b.

Mitchell, D. L., R. P. Lin, C. Mazelle, H. Rème, P. A. Cloutier, J. E. P. Connerney, M. H. Acuña, and N. F. Ness, Probing Mars' crustal magnetic field and ionosphere with the MGS Electron Reflectometer, J. Geophys. Res., 106, 23,419-23,427, 2001.

Mouginot, J., W. Kofman, A. Safaeinili, and A. Herique, Correction of the ionospheric distortion on the MARSIS surface sounding echoes, Planet. Space Sci., 57, 917-926, 2008.

Němec, F., D. D. Morgan, D. A. Gurnett, and F. Duru, Nightside ionosphere of Mars: Radar soundings by the Mars Express spacecraft, $J$. Geophys. Res., 115, E12009, 2010.

Opgenoorth, H. J., R. S. Dhillon, L. Rosenqvist, M. Lester, N. J. T. Edberg, S. E. Milan, P. Withers, and D. Brain, Day-side ionospheric conductivities at Mars, Planet. Space Sci., 58, 1139-1151, 2010.

Safaeinili, A., W. Kofman, J. Mouginot, Y. Gim, A. Herique, A. B. Ivanov, J. J. Plaut, and G. Picardi, Estimation of the total electron content of the Martian ionosphere using radar sounder surface echoes, Geophys. Res. Lett., 34, L23204, 2007.

Sheehan, C. H. and J.-P. St.-Maurice, Dissociative recombination of $\mathrm{N}_{2}^{+}$, $\mathrm{O}_{2}^{+}$, and $\mathrm{NO}^{+}$: Rate coefficients for ground state and vibrationally excited ions, J. Geophys. Res., 109, A03302, 2004.

Withers, P., M. Mendillo, H. Rishbeth, D. P. Hinson, and J. Arkani-Hamed, Ionospheric characteristics above Martian crustal magnetic anomalies, Geophys. Res. Lett., 32, L16204, 2005.

M. O. Fillingim (e-mail: matt@ssl.berkeley.edu), R. J. Lillis, S. L. England, L. M. Peticolas, D. A. Brain, J. S. Halekas, C. Paty, D. Lummerzheim, and S. W. Bougher 